\title{
Genetic and non-genetic factors affecting morphometry of Sirohi goats
}

\author{
S. D. Dudhe ${ }^{1}$, S. B. S. Yadav ${ }^{1}$, R. K. Nagda², Urmila Pannu ${ }^{1}$ and G. C. Gahlot ${ }^{1}$
}

1. Department of Animal Breeding \& Genetics, College of Veterinary and Animal Science, Bikaner - 334001 , Rajasthan, India; 2. Principal Investigator, All India Co-ordinated Research Project on Sirohi Goats, Udaipur - 313 601, Rajasthan, India.

Corresponding author: S. D. Dudhe, e-mail: drshashankdudhe@gmail.com, SBSY: yadavsbs@gmail.com, RKN: nagdark2006@yahoo.co.in, UP: urmila_pannu@yahoo.co.in, GCG: gahlotgcbkn@rediffmail.com Received: 19-06-2015, Revised: 08-10-2015, Accepted: 15-10-2015, Published online: 28-11-2015

doi: 10.14202/vetworld.2015.1356-1363 How to cite this article: Dudhe SD, Yadav SBS, Nagda RK, Pannu U, Gahlot GC (2015) Genetic and non-genetic factors affecting morphometry of Sirohi goats, Veterinary World 8(11): 1356-1363.

\begin{abstract}
Aim: The aim was to estimate genetic and non-genetic factors affecting morphometric traits of Sirohi goats under field condition.

Materials and Methods: The detailed information of all animals on body measurements at birth, 3, 6, 9, and 12 months of age was collected from farmer's flock under field condition born during 2007-2013 to analyze the effect of genetic and nongenetic factors. The least squares maximum likelihood program was used to estimate genetic and non-genetic parameters affecting morphometric traits.
\end{abstract}

Results and Discussion: Effect of sire, cluster, year of birth, and sex was found to be highly significant $(\mathrm{p}<0.01)$ on all three morphometric traits, parity was highly significant $(\mathrm{p}<0.01)$ for body height $(\mathrm{BH})$ and body girth $(\mathrm{BG})$ at birth. The $\mathrm{h}^{2}$ estimates for morphometric traits ranged among $0.528 \pm 0.163$ to $0.709 \pm 0.144$ for $\mathrm{BH}, 0.408 \pm 0.159$ to $0.605 \pm 0.192$ for body length (BL), and $0.503 \pm 0.197$ to $0.695 \pm 0.161$ for BG.

Conclusion: The effect of sire was highly significant $(\mathrm{p}<0.01)$ and also $\mathrm{h}^{2}$ estimate of all morphometric traits were medium to high; therefore, it could be concluded on the basis of present findings that animals with higher body measurements at initial phases of growth will perform better with respect to even body weight traits at later stages of growth.

Keywords: correlation, genetic, morphometric traits, non-genetic and Sirohi.

\section{Introduction}

Goat substantially contributes to the rural economy and provide livelihood to the poor sections. The total goat population of the country is 135.17 million, and it constitutes $26.46 \%$ of total livestock population.

Rajasthan ranks the first in total goat population of the country, i.e., 16.03\% [1]. Among various genotypes available the Sirohi is one of the principal and renowned breed of goats. This breed has derived its name from Sirohi district of Rajasthan [2]. The source of income from this breed depends mainly on meat and milk production. In meat producing animals like sheep and goats, external body measurements could be a reliable indicator of its future performance with respect to live body weights, if and only if a correlation has been identified among these traits of interest [3]. Growth traits, which are available early, are a very important economic trait and could serve as an indicator for improvement of the traits that appear at the later age due to the association of body weights, and body measurements with fiber production [4].

In the present study, an attempt was made to study genetic and non-genetic factors affecting the body measurements along with genetic parameters.

Copyright: The authors. This article is an open access article licensed under the terms of the Creative Commons Attributin License (http:// creative commons.org/licenses/by/2.0) which permits unrestricted use, distribution and reproduction in any medium, provided the work is properly cited.

\section{Materials and Methods}

Data

The detailed information of 3551 kids (male-1768 and female-1783) born during 2007-2013 on body measurements at birth, 3, 6, 9, and 12 months of age was collected from farmer's flock under field condition, which were maintained under All India Coordinated Research Project (AICRP) on Sirohi goats, Livestock Research Station (LRS), Vallabhnagar, Udaipur, Rajasthan. Under this project, all Sirohi breeders were identified in the field. The study area (Figure-1) is located in western part of India and situated at $582 \mathrm{~m}$ above mean sea level on $24^{\circ} 35^{\prime \prime} \mathrm{N}$ latitude and $73^{\circ} 43^{\prime \prime}$

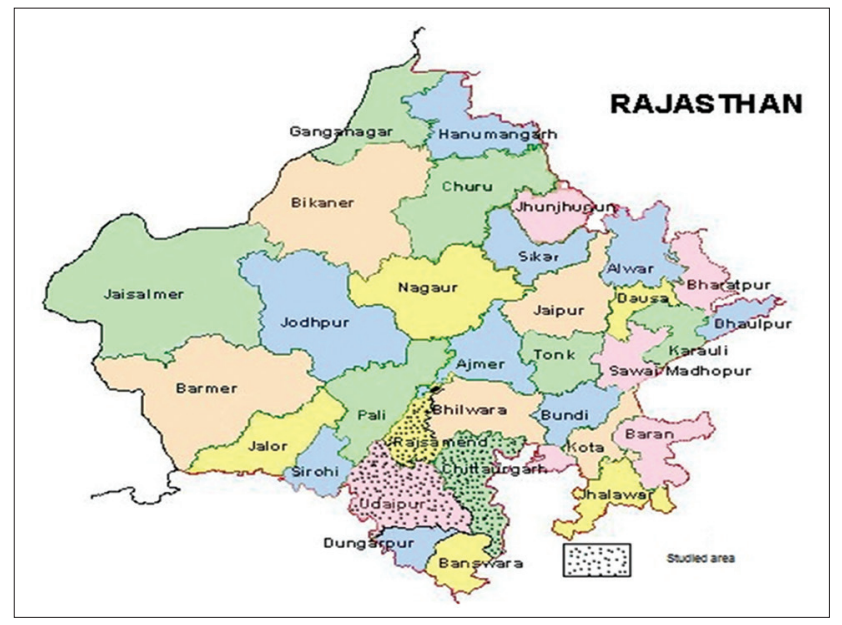

Figure-1: Study area map. 
E longitudes, which characterized with semi-arid climate with undulated topography having an average rainfall of $660 \mathrm{~mm}$ annually. Similarly, the temperature ranges from $2.3^{\circ} \mathrm{C}$ to $42.3^{\circ} \mathrm{C}$. Breeding bucks properly tagged were reared and maintained at LRS under AICRP, Vallabhnagar during off breeding season and distributed to identified farmers during breeding season. Different bucks were rotated among farmers in different breeding seasons. The kids born out of such matings were tagged, and their pedigree records were maintained at LRS, Vallabhnagar. Animals were vaccinated against enterotoxaemia and peste des petits ruminants. The data were recorded on the same day for body weight and body measurements. The records were taken from birth up to 12 months of age at the interval of 3 months by the staff members of LRS. The data were classified into five clusters of three districts viz., (1) Vallabhnagar cluster of Udaipur district, (2) Railmagra, (3) Devgarh, (4) Nathdwara clusters of Rajasamand district, and (5) Bhadsoda cluster of Chittorgarh district, three seasons of birth viz. (1) rainy (July-October), (2) winter (November-February) and (3) summer (March-June), 6 years of birth from April month of the year up to March month of next calendar year, year 1 (2007-08), year 2 (2008-09), year 3 (2009-10), year 4 (2010-11), year 5 (2011-12), and year $6(2012-13)$, five parity $(1,2,3,4$, and $\geq 5)$, two types of birth:(1) single and (2) multiple and two sex:(1) Male and (2) female.

\section{Statistical methods}

The data on growth trait was analyzed through mixed model least-squares and maximum Likelihood Computer Program PC 2, Harvey [5]. To study the effect of various genetic and non-genetic factors on body measurements the statistical model used was as under:

$$
\begin{aligned}
\mathrm{Y}_{\mathrm{ijklmnop}}= & \mu+\mathrm{a}_{\mathrm{i}}+\mathrm{B}_{\mathrm{j}}+\mathrm{C}_{\mathrm{k}}+\mathrm{D}_{1}+\mathrm{E}_{\mathrm{m}}+\mathrm{F}_{\mathrm{n}}+\mathrm{G}_{\mathrm{o}}+ \\
& \mathrm{b}\left(\mathrm{DW}_{\mathrm{ijk} k \mathrm{mnnp}}-\overline{\mathrm{DW}}\right)+\mathrm{e}_{\mathrm{ijk} k m n o p}
\end{aligned}
$$

Where,

$\mathrm{Y}_{\mathrm{ijklmnop}}=$ Performance record of the $\mathrm{p}^{\text {th }}$ progeny of $i^{\text {th }}$ sire belonging to $j^{\text {th }}$ cluster, $k^{\text {th }}$ season of birth, $1^{\text {th }}$ year of birth, $\mathrm{m}^{\text {th }}$ parity, $\mathrm{n}^{\text {th }}$ type of birth and $\mathrm{o}^{\text {th }}$ sex.

$\mu=$ Overall population mean

$a_{i}=$ Random effect of $i^{\text {th }}$ sire

$B_{j}=$ Fixed effect of $j^{\text {th }}$ cluster $(j=1,2,3,4,5)$

$\mathrm{C}_{\mathrm{k}}=$ Fixed effect of $\mathrm{k}^{\text {th }}$ season of birth $(\mathrm{k}=1,2,3)$

$\mathrm{D}_{1}=$ Fixed effect of $1^{\text {th }}$ year of birth $(1=1,2,3$,

$4,5,6)$

$\mathrm{E}_{\mathrm{m}}=$ Fixed effect of $\mathrm{m}^{\text {th }}$ parity $(\mathrm{m}=1,2,3,4, \geq 5)$

$\mathrm{F}_{\mathrm{n}}^{\mathrm{m}}=$ Fixed effect of $\mathrm{n}^{\text {th }}$ type of birth $(\mathrm{n}=1,2)$

$\mathrm{G}_{\mathrm{o}}^{\mathrm{n}}=$ Fixed effect of $\mathrm{o}^{\text {th }} \mathrm{sex}(\mathrm{o}=1,2)$

$\mathrm{b}\left(\mathrm{DW}_{\mathrm{ijk} k \mathrm{lmnop}}-\overline{\mathrm{DW}}\right)=$ The regression of the trait on dam's weight at kidding

$\mathrm{e}_{\mathrm{ijklmnop}}=$ Random error NID $\left(0, \sigma^{2}\right)$
Duncan's multiple range test as modified by Kramer [6] was used to make pairwise comparison among the least-squares means.

\section{Estimation of heritability}

The heritability was estimated by sire component of variance-covariance obtained from the paternal half-sib analysis. The standard error of heritability was estimated as per Swiger et al. [7].

\section{Genetic and phenotypic correlations}

The genetic and phenotypic correlations among traits were calculated from the analysis of variance and covariance among sire groups.

\section{Results and Discussion}

The estimates of least-squares means of body height $(\mathrm{BH})$, body length (BL) and body girth (BG) at birth, 3, 6, 9 and 12 months of ages are given in Tables-1-3, respectively.

\section{Effect of sire}

The effect of sire was found to be highly significant $(\mathrm{p}<0.01)$ on all morphometric traits at birth, 3 , 6,9 , and 12 months of age. The finding was in agreement with the observations of Kumar et al. [8] in Jamunapari goats and Karna et al. $[9,10]$ in Cheghu goats. Tomar et al. [3] reported no significant effect of sire on the three morphometric traits.

Sire significantly affected the morphometric traits at all ages indicating the existence of additive genetic variability among these traits and significant influence of sire might be attributed to relative merits of the sires used.

\section{Effect of cluster}

The effect of the cluster was found to be highly significant $(p<0.01)$ on all morphometric traits at birth, 3, 6, 9, and 12 months of ages. Sharma et al. [11] observed highly significant $(p<0.01)$ effect of cluster on BH and BL at birth and 3 months of age in Sirohi goats, BG at 3 months of age. However, Patil et al. [12] observed highly significant $(p<0.01)$ effect on $B G$ at 1 month of age, $\mathrm{BH}$ and $\mathrm{BG}$ at 6 months of age and BG at 9 months of age. Kharkar et al. [13] observed a significant effect on BL at birth. Gohain et al. [14] observed highly significant $(\mathrm{p}<0.01)$ effect of cluster on BG and BH in Assam local goats. Kuralkar et al. [15] reported highly significant $(\mathrm{p}<0.01)$ effect on all three measurements. Differences across clusters might be due to differences in grasses and herbage availability. Significantly $(p<0.01)$ higher mean body measurements were observed in Bhadsoda cluster as compared with remaining four clusters in all age groups except BL at birth.

\section{Effect of year}

The effect of year of birth was found to be highly significant $(\mathrm{p}<0.01)$ on all morphometric traits at birth, 3, 6, 9, and 12 months of ages. Present findings are similar with Tomar et al. [3] except at birth in $\mathrm{BH}$ in Sirohi goats, Sharma et al. [11] observed highly 
Table-1: Least-squares means and SE for BH (cm) of Sirohi goat at different ages.

\begin{tabular}{|c|c|c|c|c|c|}
\hline \multirow[t]{2}{*}{ Factors } & \multicolumn{5}{|c|}{ Traits } \\
\hline & At birth & 3 months & 6 months & 9 months & 12 months \\
\hline Overall mean $(\mu)$ & $\begin{array}{c}31.02 \pm 0.35 \\
(3551)\end{array}$ & $\begin{array}{c}51.53 \pm 1.19 \\
(3073)\end{array}$ & $\begin{array}{c}57.65 \pm 0.95 \\
(2194)\end{array}$ & $\begin{array}{c}61.76 \pm 2.24 \\
(1642)\end{array}$ & $\begin{array}{c}67.09 \pm 0.95 \\
(1144)\end{array}$ \\
\hline Sire & $* *$ & $* *$ & $* *$ & $* *$ & $* *$ \\
\hline Cluster & $* *$ & $* *$ & $* *$ & $* *$ & $* *$ \\
\hline Vallabhnagar & $\begin{array}{c}29.16 \pm 0.38^{\mathrm{a}} \\
(278)\end{array}$ & $\begin{array}{c}50.54 \pm 1.23^{b} \\
(214)\end{array}$ & $\begin{array}{c}55.52 \pm 1.04^{b} \\
(123)\end{array}$ & $\begin{array}{c}59.10 \pm 2.30^{\mathrm{a}} \\
(59)\end{array}$ & $\begin{array}{c}59.54 \pm 1.65^{a} \\
(36)\end{array}$ \\
\hline Railmagra & $\begin{array}{c}31.03 \pm 0.38^{b} \\
(650)\end{array}$ & $\begin{array}{c}47.35 \pm 1.22^{\mathrm{a}} \\
(593)\end{array}$ & $\begin{array}{c}57.44 \pm 1.01^{c} \\
(504)\end{array}$ & $\begin{array}{c}60.71 \pm 2.27^{b} \\
(396)\end{array}$ & $\begin{array}{c}65.82 \pm 1.10^{c} \\
(229)\end{array}$ \\
\hline Devgarh & $\begin{array}{c}31.70 \pm 0.38^{c} \\
(1584)\end{array}$ & $\begin{array}{c}52.63 \pm 1.22^{\mathrm{cb}} \\
(1439)\end{array}$ & $\begin{array}{c}57.42 \pm 1.65^{c} \\
(1184)\end{array}$ & $\begin{array}{c}61.62 \pm 2.27^{c} \\
(943)\end{array}$ & $\begin{array}{c}64.76 \pm 1.12^{\mathrm{b}} \\
(715)\end{array}$ \\
\hline Nathdwara & $\begin{array}{c}29.38 \pm 0.61^{a} \\
(37)\end{array}$ & $\begin{array}{c}51.31 \pm 1.71^{\mathrm{b}} \\
(15)\end{array}$ & $\begin{array}{c}53.42 \pm 1.65^{a} \\
(12)\end{array}$ & $\begin{array}{c}56.75 \pm 3.07^{a} \\
\text { (3) }\end{array}$ & - \\
\hline Bhadsoda & $\begin{array}{c}33.85 \pm 0.38^{d} \\
(1002)\end{array}$ & $\begin{array}{c}55.81 \pm 1.22^{d} \\
(812)\end{array}$ & $\begin{array}{c}64.47 \pm 1.03^{d} \\
(371)\end{array}$ & $\begin{array}{c}70.63 \pm 2.28^{d} \\
(241)\end{array}$ & $\begin{array}{c}78.24 \pm 1.17^{\mathrm{d}} \\
(164)\end{array}$ \\
\hline Season & $*$ & $* *$ & NS & $*$ & $* *$ \\
\hline Rainy & $\begin{array}{c}30.95 \pm 0.35^{\mathrm{a}} \\
(1303)\end{array}$ & $\begin{array}{l}51.18 \pm 1.19^{a} \\
\quad(1171)\end{array}$ & $\begin{array}{c}57.64 \pm 0.96 \\
(795)\end{array}$ & $\begin{array}{c}61.96 \pm 2.25^{b} \\
(507)\end{array}$ & $\begin{array}{c}67.61 \pm 0.96^{b} \\
(377)\end{array}$ \\
\hline Winter & $\begin{array}{c}30.96 \pm 0.35^{a} \\
(1723)\end{array}$ & $\begin{array}{c}51.78 \pm 1.19^{b} \\
(1443)\end{array}$ & $\begin{array}{c}57.59 \pm 0.96 \\
(1021)\end{array}$ & $\begin{array}{c}61.96 \pm 2.25^{b} \\
(829)\end{array}$ & $\begin{array}{c}66.99 \pm 0.95^{a} \\
(589)\end{array}$ \\
\hline Summer & $\begin{array}{c}31.16 \pm 0.36^{b} \\
(525)\end{array}$ & $\begin{array}{c}51.63 \pm 1.19^{b} \\
(459)\end{array}$ & $\begin{array}{c}57.71 \pm 0.97 \\
(378)\end{array}$ & $\begin{array}{c}61.36 \pm 2.27^{a} \\
(306)\end{array}$ & $\begin{array}{c}66.66 \pm 0.98^{a} \\
(178)\end{array}$ \\
\hline Year of birth & $* *$ & $* *$ & $* *$ & $* *$ & $* *$ \\
\hline 2007-08 & $\begin{array}{c}32.16 \pm 0.38^{d} \\
(491)\end{array}$ & $\begin{array}{c}53.21 \pm 1.22^{\mathrm{d}} \\
(453)\end{array}$ & $\begin{array}{c}59.40 \pm 1.00^{f} \\
(404)\end{array}$ & $\begin{array}{c}63.92 \pm 2.27^{d} \\
(350)\end{array}$ & $\begin{array}{c}66.28 \pm 1.03^{a} \\
(310)\end{array}$ \\
\hline 2008-09 & $\begin{array}{c}31.53 \pm 0.37^{c} \\
(586)\end{array}$ & $\begin{array}{c}50.98 \pm 1.22^{\mathrm{b}} \\
(530)\end{array}$ & $\begin{array}{c}56.17 \pm 1.00^{\mathrm{a}} \\
(412)\end{array}$ & $\begin{array}{c}61.36 \pm 2.27^{b} \\
(316)\end{array}$ & $\begin{array}{c}65.74 \pm 1.03^{a} \\
(209)\end{array}$ \\
\hline $2009-10$ & $\begin{array}{c}30.38 \pm 0.36^{a} \\
(624)\end{array}$ & $\begin{array}{c}50.54 \pm 1.20^{a} \\
(525)\end{array}$ & $\begin{array}{c}56.42 \pm 0.97^{a} \\
(394)\end{array}$ & $\begin{array}{c}59.82 \pm 2.25^{a} \\
(320)\end{array}$ & $\begin{array}{c}66.44 \pm 0.99^{b a} \\
(186)\end{array}$ \\
\hline $2010-2011$ & $\begin{array}{c}30.38 \pm 0.36^{a} \\
(531)\end{array}$ & $\begin{array}{c}51.25 \pm 1.20^{\mathrm{b}} \\
(467)\end{array}$ & $\begin{array}{c}57.24 \pm 0.98^{c} \\
(376)\end{array}$ & $\begin{array}{c}61.24 \pm 2.26^{b} \\
(308)\end{array}$ & $\begin{array}{c}67.68 \pm 1.02^{c} \\
(218)\end{array}$ \\
\hline $2011-2012$ & $\begin{array}{c}30.91 \pm 0.36^{b} \\
(668)\end{array}$ & $\begin{array}{c}51.91 \pm 1.20^{c} \\
(570)\end{array}$ & $\begin{array}{c}58.90 \pm 0.98^{e} \\
(406)\end{array}$ & $\begin{array}{c}62.86 \pm 2.26^{c} \\
(301)\end{array}$ & $\begin{array}{c}69.31 \pm 1.02^{\mathrm{d}} \\
(221)\end{array}$ \\
\hline $2012-2013$ & $\begin{array}{c}30.79 \pm 0.36^{b} \\
(651)\end{array}$ & $\begin{array}{c}51.29 \pm 1.21^{\mathrm{b}} \\
(528)\end{array}$ & $\begin{array}{l}57.74 \pm 1.00^{d} \\
(202)\end{array}$ & $\begin{array}{c}61.37 \pm 2.32^{\mathrm{b}} \\
(47)\end{array}$ & - \\
\hline Parity & $* *$ & NS & NS & NS & NS \\
\hline $1^{\text {st }}$ & $\begin{array}{c}30.97 \pm 0.36^{a} \\
(278)\end{array}$ & $\begin{array}{c}51.43 \pm 1.20 \\
(664)\end{array}$ & $\begin{array}{c}57.74 \pm 0.97 \\
(507)\end{array}$ & $\begin{array}{c}61.88 \pm 2.25 \\
(393)\end{array}$ & $\begin{array}{c}67.41 \pm 0.98 \\
(276)\end{array}$ \\
\hline $2^{\text {nd }}$ & $\begin{array}{c}31.06 \pm 0.35^{\text {ba }} \\
(650)\end{array}$ & $\begin{array}{c}51.44 \pm 1.19 \\
(589)\end{array}$ & $\begin{array}{c}57.82 \pm 0.96 \\
(455)\end{array}$ & $\begin{array}{c}61.84 \pm 2.25 \\
(348)\end{array}$ & $\begin{array}{c}67.18 \pm 0.97 \\
(243)\end{array}$ \\
\hline $3^{\text {rd }}$ & $\begin{array}{c}30.89 \pm 0.36^{a} \\
(1584)\end{array}$ & $\begin{array}{c}51.46 \pm 1.19 \\
(553)\end{array}$ & $\begin{array}{c}57.51 \pm 0.96 \\
(411)\end{array}$ & $\begin{array}{c}61.55 \pm 2.25 \\
(303)\end{array}$ & $\begin{array}{c}67.25 \pm 0.97 \\
(220)\end{array}$ \\
\hline $4^{\text {th }}$ & $\begin{array}{c}30.99 \pm 0.36^{a} \\
(37)\end{array}$ & $\begin{array}{c}51.74 \pm 1.19 \\
(461)\end{array}$ & $\begin{array}{c}57.76 \pm 0.97 \\
(317)\end{array}$ & $\begin{array}{c}61.99 \pm 2.25 \\
(218)\end{array}$ & $\begin{array}{c}66.77 \pm 0.98 \\
(152)\end{array}$ \\
\hline$\geq 5^{\text {th }}$ & $\begin{array}{c}31.20 \pm 0.35^{\text {ba }} \\
(1002)\end{array}$ & $\begin{array}{c}51.58 \pm 1.19 \\
(806)\end{array}$ & $\begin{array}{c}57.40 \pm 0.96 \\
(504)\end{array}$ & $\begin{array}{c}61.55 \pm 2.25 \\
(380)\end{array}$ & $\begin{array}{c}66.84 \pm 0.97 \\
(253)\end{array}$ \\
\hline Type of birth & $* *$ & $* *$ & $* *$ & $* *$ & $* *$ \\
\hline Single & $\begin{array}{c}32.12 \pm 0.35^{b} \\
(2080)\end{array}$ & $\begin{array}{c}52.58 \pm 1.19^{b} \\
(1842)\end{array}$ & $\begin{array}{c}58.70 \pm 0.96^{b} \\
(1386)\end{array}$ & $\begin{array}{c}62.46 \pm 2.24^{b} \\
(1049)\end{array}$ & $\begin{array}{c}67.74 \pm 0.95^{b} \\
(752)\end{array}$ \\
\hline Multiple & $\begin{array}{c}29.93 \pm 0.35^{\mathrm{a}} \\
(1471)\end{array}$ & $\begin{array}{c}50.48 \pm 1.19^{a} \\
(1231)\end{array}$ & $\begin{array}{c}56.60 \pm 0.96^{a} \\
(808)\end{array}$ & $\begin{array}{c}61.06 \pm 2.25^{a} \\
(593)\end{array}$ & $\begin{array}{c}66.44 \pm 0.96^{a} \\
(392)\end{array}$ \\
\hline Sex & $* *$ & $* *$ & $* *$ & $* *$ & $* *$ \\
\hline Male & $\begin{array}{c}31.41 \pm 0.35^{b} \\
(1768)\end{array}$ & $\begin{array}{c}52.49 \pm 1.19^{b} \\
(1536)\end{array}$ & $\begin{array}{c}58.51 \pm 0.96^{b} \\
(1023)\end{array}$ & $\begin{array}{c}62.68 \pm 2.24^{b} \\
(679)\end{array}$ & $\begin{array}{c}68.04 \pm 0.96^{b} \\
(372)\end{array}$ \\
\hline Female & $\begin{array}{c}30.64 \pm 0.35^{a} \\
(1783)\end{array}$ & $\begin{array}{c}50.57 \pm 1.19^{a} \\
(1537)\end{array}$ & $\begin{array}{c}56.78 \pm 0.96^{a} \\
(1171)\end{array}$ & $\begin{array}{c}60.84 \pm 2.24^{a} \\
(963)\end{array}$ & $\begin{array}{c}66.14 \pm 0.95^{\mathrm{a}} \\
(772)\end{array}$ \\
\hline Regression on weight of dam at kidding & $* *$ & $*$ & $*$ & NS & $* *$ \\
\hline Regression coefficient (b) $(\mathrm{kg} / \mathrm{kg})$ & $0.066 \pm 0.015$ & $0.068 \pm 0.031$ & $0.076 \pm 0.037$ & $0.046 \pm 0.048$ & $0.157 \pm 0.057$ \\
\hline
\end{tabular}

Number of observations are given in parentheses. Estimates with different superscripts differ significantly. $* * H i g h l y$ significant $(p<0.01), *$ Significant $(p<0.05), N S=$ Non-significant, $S E=$ Standard error, $\mathrm{BH}=$ Body height

significant $(\mathrm{p}<0.01)$ effect of cluster on all three morphometric traits at birth and 3 months of age in Sirohi goats and Patil et al. [12] at 1 and 3 months of age on all three morphometric traits in Sangamneri goats. Karna et al. [10] reported the significant effect on all three traits at 3 and 6 months of age in Cheghu goats.

Higher values of all three morphometric traits were observed at birth in initial 2 years (2007-2008 and 2008-2009) then it remain almost constant. The 
Table-2: Least-squares means and SE for BL $(\mathrm{cm})$ of Sirohi goat at different ages.

\begin{tabular}{|c|c|c|c|c|c|}
\hline \multirow[t]{2}{*}{ Factors } & \multicolumn{5}{|c|}{ Traits } \\
\hline & At birth & 3 months & 6 months & 9 months & 12 months \\
\hline $\begin{array}{l}\text { Overall mean } \\
(\mu)\end{array}$ & $\begin{array}{c}28.29 \pm 0.39 \\
(3551)\end{array}$ & $\begin{array}{c}47.90 \pm 1.20 \\
(3073)\end{array}$ & $\begin{array}{c}53.96 \pm 1.01 \\
(2194)\end{array}$ & $\begin{array}{c}57.36 \pm 2.16 \\
(1642)\end{array}$ & $\begin{array}{c}62.65 \pm 098 \\
(1144)\end{array}$ \\
\hline Sire & $* *$ & $* *$ & $* *$ & $* *$ & $* *$ \\
\hline Cluster & $* *$ & $* *$ & $* *$ & $* *$ & $* *$ \\
\hline Vallabhnagar & $\begin{array}{c}27.16 \pm 0.42^{\mathrm{a}} \\
(278)\end{array}$ & $\begin{array}{c}48.79 \pm 1.25^{b} \\
(214)\end{array}$ & $\begin{array}{c}54.01 \pm 1.09^{b} \\
(123)\end{array}$ & $\begin{array}{c}56.85 \pm 2.22^{\mathrm{b}} \\
(59)\end{array}$ & $\begin{array}{c}56.55 \pm 1.67^{a} \\
(36)\end{array}$ \\
\hline Railmagra & $\begin{array}{c}28.65 \pm 0.42^{b} \\
(650)\end{array}$ & $\begin{array}{c}45.63 \pm 1.24^{a} \\
(593)\end{array}$ & $\begin{array}{c}55.48 \pm 1.08^{c} \\
(504)\end{array}$ & $\begin{array}{c}59.28 \pm 2.18^{c} \\
(396)\end{array}$ & $\begin{array}{c}63.90 \pm 1.13^{c} \\
(229)\end{array}$ \\
\hline Devgarh & $\begin{array}{c}29.71 \pm 0.42^{d} \\
(1584)\end{array}$ & $\begin{array}{c}45.48 \pm 1.24^{a} \\
(1439)\end{array}$ & $\begin{array}{c}50.09 \pm 1.07^{a} \\
(1184)\end{array}$ & $\begin{array}{c}54.53 \pm 2.19^{a} \\
(943)\end{array}$ & $\begin{array}{c}58.07 \pm 1.14^{b} \\
(715)\end{array}$ \\
\hline Nathdwara & $\begin{array}{c}27.00 \pm 0.66^{a} \\
(37)\end{array}$ & $\begin{array}{c}49.78 \pm 1.76^{\mathrm{bc}} \\
\text { (15) }\end{array}$ & $\begin{array}{c}51.71 \pm 1.70^{\mathrm{a}} \\
(12)\end{array}$ & $\begin{array}{c}51.36 \pm 3.05^{a} \\
\text { (3) }\end{array}$ & - \\
\hline Bhadsoda & $\begin{array}{c}28.93 \pm 0.42^{c} \\
(1002)\end{array}$ & $\begin{array}{c}49.80 \pm 1.24^{c} \\
(812)\end{array}$ & $\begin{array}{c}58.51 \pm 1.09^{d} \\
(371)\end{array}$ & $\begin{array}{c}64.77 \pm 2.20^{d} \\
(241)\end{array}$ & $\begin{array}{c}72.07 \pm 1.19^{d} \\
(164)\end{array}$ \\
\hline Season & $* *$ & $* *$ & NS & NS & NS \\
\hline Rainy & $\begin{array}{c}28.21 \pm 0.39^{a} \\
(1303)\end{array}$ & $\begin{array}{c}47.57 \pm 1.20^{\mathrm{a}} \\
(1171)\end{array}$ & $\begin{array}{c}53.98 \pm 1.01 \\
(795)\end{array}$ & $\begin{array}{c}57.60 \pm 2.16 \\
(507)\end{array}$ & $\begin{array}{c}62.74 \pm 0.99 \\
(377)\end{array}$ \\
\hline Winter & $\begin{array}{c}28.13 \pm 0.39 a \\
(1723)\end{array}$ & $\begin{array}{c}48.06 \pm 1.20^{b} \\
(1443)\end{array}$ & $\begin{array}{c}54.12 \pm 1.02 \\
(1021)\end{array}$ & $\begin{array}{c}57.21 \pm 2.16 \\
(829)\end{array}$ & $\begin{array}{c}62.47 \pm 0.98 \\
(589)\end{array}$ \\
\hline Summer & $\begin{array}{c}28.53 \pm 0.40^{b} \\
(525)\end{array}$ & $\begin{array}{c}48.06 \pm 1.21^{\mathrm{b}} \\
(459)\end{array}$ & $\begin{array}{c}53.78 \pm 1.02 \\
(378)\end{array}$ & $\begin{array}{c}57.27 \pm 2.16 \\
(306)\end{array}$ & $\begin{array}{c}62.74 \pm 1.01 \\
(178)\end{array}$ \\
\hline Year of birth & $* *$ & $* *$ & $* *$ & $* *$ & $* *$ \\
\hline 2007-08 & $\begin{array}{c}29.62 \pm 0.42^{\mathrm{e}} \\
(491)\end{array}$ & $\begin{array}{c}49.96 \pm 1.24^{d} \\
(453)\end{array}$ & $\begin{array}{c}55.68 \pm 1.06^{\mathrm{e}} \\
(404)\end{array}$ & $\begin{array}{c}60.84 \pm 2.19^{d} \\
(350)\end{array}$ & $\begin{array}{c}64.47 \pm 1.06 \\
(310)\end{array}$ \\
\hline 2008-09 & $\begin{array}{c}28.98 \pm 0.41^{\mathrm{d}} \\
(586)\end{array}$ & $\begin{array}{c}48.08 \pm 1.23^{c} \\
(530)\end{array}$ & $\begin{array}{c}52.82 \pm 1.05^{b} \\
(412)\end{array}$ & $\begin{array}{c}56.65 \pm 2.19^{b} \\
(316)\end{array}$ & $\begin{array}{c}60.90 \pm 1.06^{a} \\
(209)\end{array}$ \\
\hline $2009-10$ & $\begin{array}{c}27.80 \pm 0.40^{\mathrm{b}} \\
(624)\end{array}$ & $\begin{array}{c}46.00 \pm 1.21^{\mathrm{a}} \\
(525)\end{array}$ & $\begin{array}{c}52.13 \pm 1.03^{a} \\
(394)\end{array}$ & $\begin{array}{c}55.25 \pm 2.17^{a} \\
(320)\end{array}$ & $\begin{array}{c}61.65 \pm 1.02^{b} \\
(186)\end{array}$ \\
\hline $2010-2011$ & $\begin{array}{c}27.42 \pm 0.40^{a} \\
(531)\end{array}$ & $\begin{array}{c}47.63 \pm 1.22^{\mathrm{b}} \\
(467)\end{array}$ & $\begin{array}{c}53.62 \pm 1.04^{c} \\
(376)\end{array}$ & $\begin{array}{c}56.50 \pm 2.17^{b} \\
(308)\end{array}$ & $\begin{array}{c}62.43 \pm 1.05^{c} \\
(218)\end{array}$ \\
\hline 2011-2012 & $\begin{array}{c}27.82 \pm 0.40^{b} \\
(668)\end{array}$ & $\begin{array}{c}48.13 \pm 1.22^{c} \\
(570)\end{array}$ & $\begin{array}{c}55.31 \pm 1.03^{e} \\
(406)\end{array}$ & $\begin{array}{c}58.20 \pm 2.17^{c} \\
(301)\end{array}$ & $\begin{array}{c}63.78 \pm 1.05^{d} \\
(221)\end{array}$ \\
\hline $2012-2013$ & $\begin{array}{c}28.09 \pm 0.41^{c} \\
(651)\end{array}$ & $\begin{array}{c}47.58 \pm 1.22^{\mathrm{b}} \\
(528)\end{array}$ & $\begin{array}{c}54.21 \pm 1.05^{d} \\
(202)\end{array}$ & $\begin{array}{c}56.72 \pm 2.24^{b} \\
(47)\end{array}$ & - \\
\hline Parity & NS & NS & NS & NS & NS \\
\hline $1^{\text {st }}$ & $\begin{array}{c}28.22 \pm 0.40 \\
(278)\end{array}$ & $\begin{array}{c}47.75 \pm 1.21 \\
(664)\end{array}$ & $\begin{array}{c}54.14 \pm 1.03 \\
(507)\end{array}$ & $\begin{array}{c}57.60 \pm 2.17 \\
(393)\end{array}$ & $\begin{array}{c}62.61 \pm 1.01 \\
(276)\end{array}$ \\
\hline $2^{\text {nd }}$ & $\begin{array}{c}28.36 \pm 0.40 \\
(650)\end{array}$ & $\begin{array}{c}47.81 \pm 1.21 \\
(589)\end{array}$ & $\begin{array}{c}54.17 \pm 1.02 \\
(455)\end{array}$ & $\begin{array}{c}57.47 \pm 2.16 \\
(348)\end{array}$ & $\begin{array}{c}62.90 \pm 1.00 \\
(243)\end{array}$ \\
\hline $3^{\text {rd }}$ & $\begin{array}{l}28.19 \pm 0.40 \\
(1584)\end{array}$ & $\begin{array}{c}47.90 \pm 1.21 \\
(553)\end{array}$ & $\begin{array}{c}53.79 \pm 1.02 \\
(411)\end{array}$ & $\begin{array}{c}57.26 \pm 2.16 \\
(303)\end{array}$ & $\begin{array}{c}62.79 \pm 1.00 \\
(220)\end{array}$ \\
\hline $4^{\text {th }}$ & $\begin{array}{c}28.32 \pm 0.40 \\
(37)\end{array}$ & $\begin{array}{c}48.12 \pm 1.21 \\
(461)\end{array}$ & $\begin{array}{c}53.96 \pm 1.02 \\
(317)\end{array}$ & $\begin{array}{c}57.36 \pm 2.17 \\
(218)\end{array}$ & $\begin{array}{c}62.54 \pm 1.01 \\
(152)\end{array}$ \\
\hline$\geq 5^{\text {th }}$ & $\begin{array}{c}28.37 \pm 0.40 \\
(1002)\end{array}$ & $\begin{array}{c}47.91 \pm 1.21 \\
(806)\end{array}$ & $\begin{array}{c}53.75 \pm 1.02 \\
(504)\end{array}$ & $\begin{array}{c}57.11 \pm 2.16 \\
(380)\end{array}$ & $\begin{array}{c}62.40 \pm 1.00 \\
(253)\end{array}$ \\
\hline Type of birth & $* *$ & $* *$ & $* *$ & $* *$ & $* *$ \\
\hline Single & $\begin{array}{c}29.37 \pm 0.39^{b} \\
(2080)\end{array}$ & $\begin{array}{c}48.83 \pm 1.20^{b} \\
(1842)\end{array}$ & $\begin{array}{c}55.06 \pm 1.01^{b} \\
(1386)\end{array}$ & $\begin{array}{c}58.07 \pm 2.16^{b} \\
(1049)\end{array}$ & $\begin{array}{c}63.20 \pm 0.98^{b} \\
(752)\end{array}$ \\
\hline Multiple & $\begin{array}{c}27.21 \pm 0.39^{a} \\
(1471)\end{array}$ & $\begin{array}{c}46.96 \pm 1.20^{\mathrm{a}} \\
(1231)\end{array}$ & $\begin{array}{c}52.86 \pm 1.02^{a} \\
(808)\end{array}$ & $\begin{array}{c}56.65 \pm 2.16^{a} \\
(593)\end{array}$ & $\begin{array}{c}62.09 \pm 0.99^{a} \\
(392)\end{array}$ \\
\hline Sex & $* *$ & $* *$ & $* *$ & $* *$ & $* *$ \\
\hline Male & $\begin{array}{c}28.62 \pm 0.39^{b} \\
(1768)\end{array}$ & $\begin{array}{c}48.71 \pm 1.20^{\mathrm{b}} \\
(1536)\end{array}$ & $\begin{array}{l}54.72 \pm 1.01^{\mathrm{b}} \\
(1023)\end{array}$ & $\begin{array}{c}58.32 \pm 2.16^{b} \\
(679)\end{array}$ & $\begin{array}{c}63.65 \pm 0.99^{b} \\
(372)\end{array}$ \\
\hline Female & $\begin{array}{c}27.96 \pm 0.39^{a} \\
(1783)\end{array}$ & $\begin{array}{c}47.08 \pm 1.20^{a} \\
(1537)\end{array}$ & $\begin{array}{c}53.20 \pm 1.01^{a} \\
(1171)\end{array}$ & $\begin{array}{c}56.40 \pm 2.16^{a} \\
(963)\end{array}$ & $\begin{array}{c}61.65 \pm 0.98^{a} \\
(772)\end{array}$ \\
\hline Regression on weight of dam at kidding & $* *$ & NS & * & NS & $* *$ \\
\hline Regression coefficient (b) $(\mathrm{kg} / \mathrm{kg})$ & $0.067 \pm 0.016$ & $0.027 \pm 0.033$ & $0.089 \pm 0.038$ & $0.062 \pm 0.050$ & $0.152 \pm 0.057$ \\
\hline
\end{tabular}

Number of observations are given in parentheses. Estimates with different superscripts differ significantly. $* * H i g h l y$ significant $(p<0.01)$, *Significant $(p<0.05)$. NS=Non-significant, SE=Standard error, BL=Body length

$\mathrm{BH}$ declined upto $2^{\text {nd }}$ year and then again remained unchanged.The BG continuously declined up to 6 and 9 months of age. The highest BL was observed in the $1^{\text {st }}$ year of birth (2007-2008), whereas BH and BG in last year of birth (2012-2013). This might be due to the differences in climate, nutrition and management.

\section{Effect of season of birth}

Influence of season of birth was highly significant $(\mathrm{p}<0.01)$ on $\mathrm{BH}$ at 3 and 12 months of ages, 
Table-3: Least-squares means and SE for BG (cm) of Sirohi goat at different ages.

\begin{tabular}{|c|c|c|c|c|c|}
\hline \multirow[t]{2}{*}{ Factors } & \multicolumn{5}{|c|}{ Traits } \\
\hline & At birth & 3 months & 6 months & 9 months & 12 months \\
\hline $\begin{array}{l}\text { Overall mean } \\
(\mu)\end{array}$ & $\begin{array}{c}31.19 \pm 0.41 \\
(3551)\end{array}$ & $\begin{array}{c}51.62 \pm 1.18 \\
(3073)\end{array}$ & $\begin{array}{c}58.64 \pm 1.00 \\
(2194)\end{array}$ & $\begin{array}{c}62.26 \pm 2.35 \\
(1642)\end{array}$ & $\begin{array}{c}67.51 \pm 1.06 \\
(1144)\end{array}$ \\
\hline Sire & $* *$ & $* *$ & $* *$ & $* *$ & $* *$ \\
\hline Cluster & $* *$ & $* *$ & $* *$ & $* *$ & $* *$ \\
\hline Vallabhnagar & $\begin{array}{c}29.69 \pm 0.44^{b} \\
(278)\end{array}$ & $\begin{array}{c}51.20 \pm 1.22^{\mathrm{b}} \\
(214)\end{array}$ & $\begin{array}{c}56.08 \pm 1.09^{a} \\
(123)\end{array}$ & $\begin{array}{c}59.90 \pm 2.40^{\mathrm{a}} \\
(59)\end{array}$ & $\begin{array}{c}59.66 \pm 1.73^{a} \\
(36)\end{array}$ \\
\hline Railmagra & $\begin{array}{c}32.25 \pm 0.44^{d} \\
(650)\end{array}$ & $\begin{array}{c}48.82 \pm 1.21^{a} \\
(593)\end{array}$ & $\begin{array}{c}59.18 \pm 1.07 c \\
(504)\end{array}$ & $\begin{array}{c}62.45 \pm 2.37 c \\
(396)\end{array}$ & $\begin{array}{c}68.36 \pm 1.20^{\circ} \\
(229)\end{array}$ \\
\hline Devgarh & $\begin{array}{c}32.02 \pm 0.44^{c} \\
(1584)\end{array}$ & $\begin{array}{c}52.90 \pm 1.21^{c} \\
(1439)\end{array}$ & $\begin{array}{c}57.72 \pm 1.06^{b} \\
(1184)\end{array}$ & $\begin{array}{c}61.84 \pm 2.37^{b} \\
(943)\end{array}$ & $\begin{array}{c}65.24 \pm 1.22^{b} \\
(715)\end{array}$ \\
\hline Nathdwara & $\begin{array}{c}29.03 \pm 0.66^{a} \\
(37)\end{array}$ & $\begin{array}{c}50.34 \pm 1.70^{\mathrm{ab}} \\
(15)\end{array}$ & $\begin{array}{c}56.81 \pm 1.77^{\mathrm{ab}} \\
(12)\end{array}$ & $\begin{array}{c}57.43 \pm 3.15^{\mathrm{a}} \\
\text { (3) }\end{array}$ & - \\
\hline Bhadsoda & $\begin{array}{c}32.98 \pm 0.43^{e} \\
(1002)\end{array}$ & $\begin{array}{c}54.84 \pm 1.21^{\mathrm{d}} \\
(812)\end{array}$ & $\begin{array}{c}63.43 \pm 1.08^{d} \\
(371)\end{array}$ & $\begin{array}{c}69.71 \pm 2.38^{d} \\
(241)\end{array}$ & $\begin{array}{c}76.77 \pm 1.26^{d} \\
(164)\end{array}$ \\
\hline Season & $* *$ & $* *$ & NS & NS & $* *$ \\
\hline Rainy & $\begin{array}{c}31.20 \pm 0.41^{\mathrm{b}} \\
(1303)\end{array}$ & $\begin{array}{c}51.24 \pm 1.18^{a} \\
(1171)\end{array}$ & $\begin{array}{c}58.54 \pm 1.00 \\
(795)\end{array}$ & $\begin{array}{c}62.33 \pm 2.35 \\
(507)\end{array}$ & $\begin{array}{c}67.93 \pm 1.07^{b} \\
(377)\end{array}$ \\
\hline Winter & $\begin{array}{c}31.02 \pm 0.41^{a} \\
(1723)\end{array}$ & $\begin{array}{c}51.85 \pm 1.18^{b} \\
(1443)\end{array}$ & $\begin{array}{c}58.63 \pm 1.00 \\
(1021)\end{array}$ & $\begin{array}{c}62.50 \pm 2.35 \\
(829)\end{array}$ & $\begin{array}{c}67.13 \pm 1.06^{a} \\
(589)\end{array}$ \\
\hline Summer & $\begin{array}{c}31.36 \pm 0.42^{\mathrm{b}} \\
(525)\end{array}$ & $\begin{array}{c}51.77 \pm 1.19^{b} \\
(459)\end{array}$ & $\begin{array}{c}58.76 \pm 1.01 \\
(378)\end{array}$ & $\begin{array}{c}61.96 \pm 2.35 \\
(306)\end{array}$ & $\begin{array}{c}67.46 \pm 1.08^{a} \\
(178)\end{array}$ \\
\hline Year of birth & $* *$ & $* *$ & $* *$ & $* *$ & $* *$ \\
\hline 2007-08 & $\begin{array}{c}32.82 \pm 0.43^{d} \\
(491)\end{array}$ & $\begin{array}{c}53.85 \pm 1.21^{\mathrm{d}} \\
(453)\end{array}$ & $\begin{array}{c}60.62 \pm 1.05^{e} \\
(404)\end{array}$ & $\begin{array}{c}65.01 \pm 2.37^{d} \\
(350)\end{array}$ & $\begin{array}{c}67.34 \pm 1.14^{b} \\
(310)\end{array}$ \\
\hline 2008-09 & $\begin{array}{c}31.95 \pm 0.43^{c} \\
(586)\end{array}$ & $\begin{array}{c}51.31 \pm 1.21^{\mathrm{b}} \\
(525)\end{array}$ & $\begin{array}{c}57.80 \pm 1.05^{a} \\
(412)\end{array}$ & $\begin{array}{c}62.58 \pm 2.37^{c} \\
(316)\end{array}$ & $\begin{array}{c}66.02 \pm 1.13^{\mathrm{a}} \\
(209)\end{array}$ \\
\hline $2009-10$ & $\begin{array}{c}30.37 \pm 0.42^{a} \\
(624)\end{array}$ & $\begin{array}{c}50.60 \pm 1.19^{a} \\
(530)\end{array}$ & $\begin{array}{c}57.37 \pm 1.01^{\mathrm{a}} \\
(394)\end{array}$ & $\begin{array}{c}60.24 \pm 2.36^{a} \\
(320)\end{array}$ & $\begin{array}{c}66.90 \pm 1.10^{b} \\
(186)\end{array}$ \\
\hline $2010-2011$ & $\begin{array}{c}30.79 \pm 0.42^{\mathrm{b}} \\
(531)\end{array}$ & $\begin{array}{c}51.19 \pm 1.19^{b} \\
(467)\end{array}$ & $\begin{array}{c}57.85 \pm 1.02^{\mathrm{ba}} \\
(376)\end{array}$ & $\begin{array}{c}61.33 \pm 2.36^{b} \\
(308)\end{array}$ & $\begin{array}{c}67.89 \pm 1.12^{\mathrm{cb}} \\
(218)\end{array}$ \\
\hline $2011-2012$ & $\begin{array}{c}30.72 \pm 0.42^{b} \\
(668)\end{array}$ & $\begin{array}{c}51.67 \pm 1.19^{c} \\
(570)\end{array}$ & $\begin{array}{c}59.68 \pm 1.02^{d} \\
(406)\end{array}$ & $\begin{array}{c}62.92 \pm 2.36^{c} \\
(301)\end{array}$ & $\begin{array}{c}69.40 \pm 1.13^{d} \\
(221)\end{array}$ \\
\hline $2012-2013$ & $\begin{array}{c}30.50 \pm 0.42^{a} \\
(651)\end{array}$ & $\begin{array}{c}51.09 \pm 1.20^{b} \\
(528)\end{array}$ & $\begin{array}{c}58.55 \pm 1.04^{c} \\
(202)\end{array}$ & $\begin{array}{c}61.51 \pm 2.42^{b} \\
(47)\end{array}$ & - \\
\hline Parity & $* *$ & NS & NS & NS & NS \\
\hline $1^{\text {st }}$ & $\begin{array}{c}31.06 \pm 0.42^{\mathrm{a}} \\
(278)\end{array}$ & $\begin{array}{c}51.47 \pm 1.19 \\
(664)\end{array}$ & $\begin{array}{c}58.63 \pm 1.01 \\
(507)\end{array}$ & $\begin{array}{c}62.17 \pm 2.36 \\
(393)\end{array}$ & $\begin{array}{c}67.69 \pm 1.09 \\
(276)\end{array}$ \\
\hline $2^{\text {nd }}$ & $\begin{array}{c}31.22 \pm 0.42^{\mathrm{a}} \\
(650)\end{array}$ & $\begin{array}{c}51.44 \pm 1.18 \\
(589)\end{array}$ & $\begin{array}{c}58.73 \pm 1.01 \\
(455)\end{array}$ & $\begin{array}{c}62.31 \pm 2.35 \\
(348)\end{array}$ & $\begin{array}{c}67.64 \pm 1.08 \\
(243)\end{array}$ \\
\hline $3^{\text {rd }}$ & $\begin{array}{c}31.11 \pm 0.42^{\mathrm{a}} \\
(1584)\end{array}$ & $\begin{array}{c}51.58 \pm 1.18 \\
(553)\end{array}$ & $\begin{array}{c}58.57 \pm 1.01 \\
(411)\end{array}$ & $\begin{array}{c}62.09 \pm 2.35 \\
(303)\end{array}$ & $\begin{array}{c}67.48 \pm 1.08 \\
(220)\end{array}$ \\
\hline $4^{\text {th }}$ & $\begin{array}{c}31.19 \pm 0.42^{\mathrm{a}} \\
(37)\end{array}$ & $\begin{array}{c}51.84 \pm 1.19 \\
(461)\end{array}$ & $\begin{array}{c}58.88 \pm 1.01 \\
(317)\end{array}$ & $\begin{array}{c}62.65 \pm 2.36 \\
(218)\end{array}$ & $\begin{array}{c}67.32 \pm 1.09 \\
(152)\end{array}$ \\
\hline$\geq 5^{\text {th }}$ & $\begin{array}{c}31.38 \pm 0.41^{\text {ba }} \\
(1002)\end{array}$ & $\begin{array}{c}51.76 \pm 1.18 \\
(806)\end{array}$ & $\begin{array}{c}58.41 \pm 1.01 \\
(504)\end{array}$ & $\begin{array}{c}62.11 \pm 2.35 \\
(380)\end{array}$ & $\begin{array}{c}67.40 \pm 1.08 \\
(253)\end{array}$ \\
\hline Type of birth & $* *$ & $* *$ & $* *$ & $* *$ & $* *$ \\
\hline Single & $\begin{array}{c}32.27 \pm 0.41^{b} \\
(2080)\end{array}$ & $\begin{array}{c}52.70 \pm 1.18^{b} \\
(1842)\end{array}$ & $\begin{array}{c}59.67 \pm 1.00^{\mathrm{b}} \\
(1386)\end{array}$ & $\begin{array}{c}62.89 \pm 2.35^{b} \\
(1049)\end{array}$ & $\begin{array}{c}68.25 \pm 1.06^{b} \\
(752)\end{array}$ \\
\hline Multiple & $\begin{array}{c}30.11 \pm 0.41^{\mathrm{a}} \\
(1471)\end{array}$ & $\begin{array}{c}50.53 \pm 1.18^{\mathrm{a}} \\
(1231)\end{array}$ & $\begin{array}{c}57.61 \pm 1.00^{\mathrm{a}} \\
(808)\end{array}$ & $\begin{array}{c}61.64 \pm 2.35^{a} \\
(593)\end{array}$ & $\begin{array}{c}66.77 \pm 1.07^{a} \\
(392)\end{array}$ \\
\hline Sex & $* *$ & $* *$ & $* *$ & $* *$ & $* *$ \\
\hline Male & $\begin{array}{c}31.58 \pm 0.41^{b} \\
(1768)\end{array}$ & $\begin{array}{c}52.55 \pm 1.18^{b} \\
(1536)\end{array}$ & $\begin{array}{c}59.52 \pm 1.00^{b} \\
(1023)\end{array}$ & $\begin{array}{c}63.18 \pm 2.35^{b} \\
(679)\end{array}$ & $\begin{array}{c}68.46 \pm 1.07^{b} \\
(372)\end{array}$ \\
\hline Female & $\begin{array}{c}30.80 \pm 0.41^{a} \\
(1783)\end{array}$ & $\begin{array}{c}50.69 \pm 1.18^{a} \\
(1537)\end{array}$ & $\begin{array}{c}57.77 \pm 1.00^{\mathrm{a}} \\
(1171)\end{array}$ & $\begin{array}{c}61.35 \pm 2.35^{a} \\
(963)\end{array}$ & $\begin{array}{c}66.56 \pm 1.06^{a} \\
(772)\end{array}$ \\
\hline Regression on weight of dam at kidding & $*$ & $*$ & NS & NS & $* *$ \\
\hline Regression coefficient (b) $(\mathrm{kg} / \mathrm{kg})$ & $0.039 \pm 0.015$ & $0.071 \pm 0.031$ & $0.037 \pm 0.041$ & $0.008 \pm 0.048$ & $0.153 \pm 0.058$ \\
\hline
\end{tabular}

Number of observations are given in parentheses. Estimates with different superscripts differ significantly. $* * H i g h l y$ significant $(p<0.01)$, *Significant $(p<0.05)$. NS=Non-significant, SE=Standard error, $B G=$ Body girth

significant at birth and 9 months of ages and non-significant at 6 months of age. Highly significant $(p<0.01)$ effect was observed on BG at birth, 3 and 12 months of ages, also on BL at birth and 3 months of ages. Similar results were also observed by Pathodiya et al. [16] in Sirohi goats. However, Sharma et al. [11] reported significant $(p<0.01)$ effect of season of birth on $\mathrm{BH}$ and $\mathrm{BL}$ at birth, highly significant $(\mathrm{p}<0.01)$ effect on all three morphometric traits at 3 months of age. Barhat [17] reported highly significant $(\mathrm{p}<0.01)$ 
effect on all three morphometric traits in Marwari goats, Kharkar et al. [13] at 3 months on BL, 3 and 12 months $\mathrm{BH}$ and at birth and 12 months $\mathrm{BG}$ on Berari goats.

Summer born kids at birth have higher BH, BL and $\mathrm{BG}$ compared with rainy season and winter season. Kids born in rainy season attended maximum $\mathrm{BH}$ and $\mathrm{BG}$.

\section{Effect of sex of kids}

Sex of kids had highly significant effect $(\mathrm{p}<0.01)$ on all three morphometric traits at birth, 3, 6, 9 and 12 months of ages. However, the male kids were larger to females one with regards to their $\mathrm{BH}, \mathrm{BL}$, and BG at all the ages. These results are in agreement with the findings of Pathodiya et al. [16], Sharma et al. [11] reported highly significant $(\mathrm{p}<0.01)$ effect of sex of kid on BL and BG at birth in Sirohi goats and Reotheia et al. [18] reported highly significant $(p<0.01)$ effect of sex of kid on BH and BG at 3 months of age and all three morphometric traitsat 6 and 9 months of age in Bakerwali goats. However, the non-significant effect of sex of kids on $\mathrm{BH}, \mathrm{BL}$ and BG on all ages was observed by Kharkar et al. [13], whereas Kharkar et al. [19] significant effect on boy height at 12 months of age in Berari goats. Gohain et al. [14] observed highly significant $(\mathrm{p}<0.01)$ effect of sex of kid on all three morphometric traits in Assam local goats.

\section{Effect of type of birth}

The type of birth had highly significant effect $(\mathrm{p}<0.01)$ on all three morphometric traits at birth, 3 , 6,9 , and 12 months of ages. These findings were in agreement to those of Tomar et al. [3] at birth, 3 and 6 months of age in Sirohi goats and Patil et al. [12] at 1 and 3 months of age in Sangamneri goats on all three morphometric traits. Sharma et al. [11] reported highly significant effect $(\mathrm{p}<0.01)$ of type of birth on all three morphometric traits at birth, significant effect $(p<0.01)$ on BH at 3 months of age. However, non-significant effect of type of birth on $\mathrm{BH}, \mathrm{BL}$ and BG on all ages was observed by Kharkar et al. [13] in Berari goats.

Single born kids were larger in BH, BL and BG than those born as multiple at all the ages. This might be due to availability of more nutrients to the single born kid than those born in multiple births during pre and postnatal life.

\section{Effect of parity}

The parity of dam had highly significant effect $(\mathrm{p}<0.01)$ on BH and BG at birth and at other ages non-significant effect of parity was observed. BL had non-significant effect of parity on all ages in the present study. Similar effect of dam's parity on morphometric traits at all ages was reported by Nahardeka et al. [20] in Assam local goats. However, Pathodiya et al. [16] reported significant effect of parity of dam on all morphometric traits at birth in Sirohi goats.

\section{Effect of dam's weight at kidding}

The regression of dam's weight at kidding had significant effect on $\mathrm{BH}$ at birth, 3, 6 and 12 months of ages, BL at birth, 6 and 12 months of ages and BG at birth, 3 and 12 months of ages. However, Kumar et al. [8] reported non-significant effect of dam's weight at kidding on all morphometric traits at 6 , 9, and 12 months of ages in Jamunapari goats and Sharma et al. [11] also reported non-significant effect on all morphometric traits at birth and 3 months of age.

\section{Genetic and phenotypic parameters for Morphometric traits at different ages}

The results regarding estimated genetic and phenotypic parameters viz. heritability, genetic and phenotypic correlations of a population are presented in Tables-4-6.

\section{Heritability}

The heritability estimates for morphometric traits under study were of high magnitude. The heritability estimates for morphometric traits ranged among $0.528 \pm 0.163$ to $0.709 \pm 0.144$ for $\mathrm{BH}, 0.408 \pm 0.159$ to $0.605 \pm 0.192$ for $\mathrm{BL}$, and $0.503 \pm 0.197$ to $0.695 \pm 0.161$ for BG. Higher estimates were also reported by Tomar et al. [3] at 3 and 6 months of age and Pathodiya et al. [16] at birth in Sirohi goats. On the other hand low heritability for BL and $\mathrm{BH}$ at 3 months and moderate heritability for morphometric traits at birth in Sirohi goats was reported by Tomar et al. [3]. The results indicated the presence of additive genetic variability and hence mass selection would be effective to improve these traits.

\section{Genetic correlation}

Estimates of genetic correlations between BHs at different ages ranged from $0.520 \pm 0.030$ for $6-9 \mathrm{BH}$ to $0.775 \pm 0.014$ for $9-12 \mathrm{BH}$, between BLs at different ages ranged from $0.478 \pm 0.126$ for BBL-6BL to $0.863 \pm 0.050$ for $6-12 \mathrm{BL}$ and between BGs at different ages ranged from $0.508 \pm 0.061$ for BBG-9BG to $0.900 \pm 0.044$ for $6-12 B G$.

\section{Phenotypic correlation}

Phenotypic correlation is the association between phenotypic values of different traits measured on the same animal. It is a joint function of the genotype, and environment and interaction if any, between the two, but their relative contributions are varied. The estimates of phenotypic correlations between different morphometric traits at different ages are presented in Tables-4-6 and are discussed as follows.

Phenotypic correlations between BHs at different ages ranged from $0.333 \pm 0.026$ for $\mathrm{BBH}-12 \mathrm{BH}$ to $0.741 \pm 0.013$ for $9-12 \mathrm{BH}$, between BLs at different ages ranged from $0.227 \pm 0.028$ for BBL-6BL to $0.703 \pm 0.015$ for $6-9 \mathrm{BL}$ and between BGs at different ages ranged from $0.340 \pm 0.026$ for BBG-6BG to $0.668 \pm 0.017$ for $9-12 B G$. 
Table-4: Estimates of heritability (on diagonal), genetic correlation (above diagonal) and phenotypic correlation (below diagonal) among $\mathrm{BH}$ at different ages in Sirohi goats.

\begin{tabular}{lccccc}
\hline Trait & BBH & 3 BH & $\mathbf{6 ~ B H}$ & $\mathbf{9} \mathbf{B H}$ & $\mathbf{1 2}$ BH \\
\hline $\mathrm{BBH}$ & $0.693 \pm 0.130$ & $0.621 \pm 0.059$ & $0.533 \pm 0.102$ & $0.636 \pm 0.080$ & $0.767 \pm 0.074$ \\
$3 \mathrm{BH}$ & $0.443 \pm 0.024$ & $0.528 \pm 0.163$ & $0.664 \pm 0.046$ & $0.691 \pm 0.040$ & $0.513 \pm 0.036$ \\
$6 \mathrm{BH}$ & $0.354 \pm 0.026$ & $0.658 \pm 0.017$ & $0.709 \pm 0.144$ & $0.520 \pm 0.030$ & $0.664 \pm 0.049$ \\
$9 \mathrm{BH}$ & $0.357 \pm 0.026$ & $0.573 \pm 0.020$ & $0.727 \pm 0.014$ & $0.699 \pm 0.179$ & $0.775 \pm 0.014$ \\
$12 \mathrm{BH}$ & $0.333 \pm 0.026$ & $0.495 \pm 0.022$ & $0.557 \pm 0.020$ & $0.741 \pm 0.013$ & $0.708 \pm 0.188$ \\
\hline
\end{tabular}

$\mathrm{BBH}=$ Body height at birth, $3 \mathrm{BH}=3$ months body height, $6 \mathrm{BH}=6$ months body height, $9 \mathrm{BH}=9$ months body height, $12 \mathrm{BH}=12$ months body height

Table-5: Estimates of heritability (on diagonal), genetic correlation (above diagonal) and phenotypic correlation (below diagonal) among BL at different ages in Sirohi goats.

\begin{tabular}{lcccrr}
\hline Trait & BBL & 3 BL & $\mathbf{6 ~ B L}$ & $\mathbf{9 ~ B L}$ & $\mathbf{1 2}$ BL \\
\hline BBL & $0.568 \pm 0.137$ & $0.584 \pm 0.069$ & $0.478 \pm 0.126$ & $0.576 \pm 0.109$ & $0.679 \pm 0.090$ \\
$3 \mathrm{BL}$ & $0.373 \pm 0.025$ & $0.408 \pm 0.159$ & $0.660 \pm 0.050$ & $0.550 \pm 0.052$ & $0.648 \pm 0.030$ \\
$6 \mathrm{BL}$ & $0.227 \pm 0.028$ & $0.555 \pm 0.020$ & $0.589 \pm 0.150$ & $0.731 \pm 0.028$ & $0.863 \pm 0.050$ \\
$9 \mathrm{BL}$ & $0.261 \pm 0.028$ & $0.513 \pm 0.022$ & $0.703 \pm 0.015$ & $0.571 \pm 0.172$ & $0.835 \pm 0.026$ \\
$12 \mathrm{BL}$ & $0.294 \pm 0.027$ & $0.458 \pm 0.023$ & $0.520 \pm 0.022$ & $0.663 \pm 0.017$ & $0.605 \pm 0.192$ \\
\hline
\end{tabular}

$\mathrm{BBL}=$ Body length at birth, $3 \mathrm{BL}=3$ months body length, $6 \mathrm{BL}=6$ months body length, $9 \mathrm{BL}=9$ months body length, $12 \mathrm{BL}=12$ months body length

Table-6: Estimates of heritability (on diagonal), genetic correlation (above diagonal) and phenotypic correlation (below diagonal) among BG at different ages in Sirohi goats.

\begin{tabular}{lccccc}
\hline Trait & BBG & 3 BG & 6 BG & 9 BG & 12 BG \\
\hline BBG & $0.590 \pm 0.147$ & $0.519 \pm 0.058$ & $0.620 \pm 0.088$ & $0.508 \pm 0.061$ & $0.608 \pm 0.061$ \\
3 BG & $0.465 \pm 0.023$ & $0.695 \pm 0.161$ & $0.582 \pm 0.047$ & $0.611 \pm 0.035$ & $0.591 \pm 0.041$ \\
6 BG & $0.340 \pm 0.026$ & $0.559 \pm 0.020$ & $0.563 \pm 0.138$ & $0.667 \pm 0.023$ & $0.900 \pm 0.044$ \\
9 BG & $0.418 \pm 0.024$ & $0.573 \pm 0.020$ & $0.645 \pm 0.017$ & $0.676 \pm 0.184$ & $0.863 \pm 0.017$ \\
12 BG & $0.394 \pm 0.025$ & $0.506 \pm 0.022$ & $0.497 \pm 0.022$ & $0.668 \pm 0.017$ & $0.503 \pm 0.197$ \\
\hline
\end{tabular}

$\mathrm{BBG}=$ Body girth at birth, $3 \mathrm{BG}=3$ months body girth, 6 BG=6 months body girth, 9 BG=9 months body girth,

$12 \mathrm{BG}=12$ months body girth

\section{Conclusion}

The effect of sire was highly significant $(\mathrm{p}<0.01)$ and also $\mathrm{h}^{2}$ estimate of all morphometric traits were medium to high; therefore, it could be concluded on the basis of present findings that animals with higher body measurements at initial phases of growth will perform better with respect to even body weight traits at later stages of growth. The estimates of phenotypic and genetic correlations were quite high in magnitude suggesting that improvement in $\mathrm{BH}$ would result in desired gain in body weight and body girth also.

\section{Authors' Contributions}

SBSY and RKN designed the experiment. SDD conducted the study and analyzed the data. UP and GCG revised the manuscript. All authors read and approved the final manuscript.

\section{Acknowledgments}

The authors are thankful to the Vice-Chancellor, Rajasthan University of Veterinary and Animal Sciences and Principal Investigator, AICRP on Sirohi goats for providing all kind of facilities and other information for this study.

\section{Competing Interests}

interests.

The authors declare that they have no competing

\section{References}

1. $19^{\text {th }}$ Livestock Census. (2012) Available from: http://www. dahd.nic.in/dahd/statistics/livestock-census.aspx. Accessed on 24-05-2015.

2. Meel, U.K., Nagda, R.K., Sharma, S.K. and Rajawat, B.S. (2010) Growth performance of Sirohi goats under field conditions. Indian J. Small Rumin., 16(1): 246-248.

3. Tomar, A.K.S., Kumar, A., Mehta, B.S. and Jaishankar, J. (2001) Factors affecting early express body traits in Sirohi goats. Indian J. Anim. Sci., 71(3): 271-273.

4. Karna, D.K., Koul, G.L. and Bisht, G.S. (2001a) Pashmina yield and its association with morphometric traits in Indian cheghu goats. Small Rumin. Res., 41: 271-275.

5. Harvey, W.R. (1990) User's Guide for LSMLMW, Mixed Model Least-Squares and Maximum Likelihood Computer Programme. Ohio State University, Columbus, Ohio, USA.

6. Kramer, C.R. (1957) Extension of multiple range tests to group correlated means. Biometrics, 13: 13-18.

7. Swiger, L.A., Harvey, W.R., Everson, D.O. and Gregory, K.E. (1964) The variance of interclass correlation involving group with one observation. Biometrics, 20: 818-826.

8. Kumar, S., Bhat, P.N., Bhat, P.P. and Palia, S.K. (1992) Genetic and non-genetic factors affecting body weights and measurements in Jamunapari goats. Indian J. Anim. Sci., 62(9): 894-897.

9. Karna, D.K., Koul, G.L. and Bisht, G.S. (2001b) Birth 
weight, morphometry and relative gain in cheghu kids. Indian J. Anim. Sci., 71(2): 180-182.

10. Karna, D.K., Bisht, G.S. and Koul, G.L. (2005) Genetic and non-genetic factors affecting morphometry of cheghu goats. Indian J. Small Rumin., 11(1): 62-64.

11. Sharma, M.C., Pathodiya, O.P., Tailor, S.P. and Mishra, S. (2010) Pre-weaning growth traits of Sirohi kids under farmer's management system. Indian J. Small Rumin., 16(1): 243-245.

12. Patil, B.S., Pachpute, S.T. and Dhage, S.A. (2013) Growth performance of Sangamneri goats under field conditions. Indian J. Small Rumin., 192: 151-155.

13. Kharkar, K., Kuralkar, S.V., Kuralkar, P., Bankar, P.S., Chopade, M.M. and Hadole, K.A. (2014) Factors affecting body weight and morphometric characters of Berari goats. Indian J. Small Rumin., 20(2): 112-114.

14. Gohain, M., Konwar, P. and Nahardeka, N. (2014) Influence of non-genetic factors on body weight and linear body measurements of Assam local goats. Indian J. Small Rumin., 20(2): 115-117.
15. Kuralkar, S.V., Varma, N.V., Kharkar, K. and Kuralkar, P. (2013) Berari goats: Characterization, management, performance and population status. Indian J. Anim. Sci., 83(12): 1292-1298.

16. Pathodiya, O.P., Gurjar, M.L., Sharma, M.C. and Khadda, B.S. (2004) Studies on birth weight and morphometry of Sirohi kids in farmers field. Indian J. Small Rumin., 10(1): 74-76.

17. Barhat, N.K. (2005) Boy weight and measurements in Marwari goats. Indian J. Anim. Sci., 75(11): 1323-1325.

18. Reotheia, A., Khan, A. and Suri, S. (2013) Morpho-metric traits of Bakerwali goats under migratory production system. Indian Vet. J., 90(12): 22-25.

19. Kharkar, K., Kuralkar, S.V. and Kuralkar, P. (2014) Growth, production and reproduction performance of Berari goats in their native tract. Indian J. Small Rumin., 20(1): 12-15.

20. Nahardeka, N., Das, D., Roy, T.C., Goswami, R.N., Das, G.C., Gopal, P.K. and Das, B. (2001) Studies on body weights of Assam local goats and their crosses with Beetal. Indian Vet. J., 78(9): 811-814. 